\title{
Rastreo de cáncer colorrectal: Análisis de costo efectividad
}

Cost-Effectiveness of Colonoscopy in Screening for Colorectal Cancer. Sonnenberg A, Delcò F, Inadomi JM. Ann. Intern Med 2000; 133: 573584.

\section{Objetivo \\ Comparar la costo efectividad de la búsqueda de sangre oculta en materia fecal, la sigmoidoscopia flexible y la colonoscopía en} el screening del cáncer colorrectal.

\section{Diseño}

Análisis de costo-efectividad. Modelo de simulación de Markov.*

\section{Lugar}

Estudio poblacional en EE. UU..

\section{Pacientes}

Mayores de 50 años de la población general

\section{Intervención}

Se realizó una simulación con una población teórica de 100.000 personas, en las que se evaluaron las siguientes estrategias: búsqueda de sangre oculta en materia fecal (SOMF) anual, sigmoidoscopia flexible cada 5 años o colonoscopía cada 10 años. Los pacientes con pruebas positivas de SOMF o pólipos adenomatosos en la sigmoidoscopía serían posteriormente explorados con colonoscopía. La colonoscopía era repetida cada 3 años tras la polipectomía, hasta que no fueran hallados pólipos.

Medición de costos y resultados Principales

Tasa de costo-efectividad incremental ${ }^{*}$

\section{Resultados Principales}

Si bien el screening con SOMF comparado con la colonoscopía tiene menor costo resulta en una menor cantidad de años de vida salvados. La estrategia de screening con sigmoidoscopia flexible cada 5 años es menos costo-efectiva que cualquiera de las otras estrategias.

La estrategia con colonoscopía es capaz de prevenir el $75 \%$ de los casos de cáncer colorrectal (7.952 años de vida salvados), mientras que la SOMF consiguió prevenir el 16\% (1.896 años de vida salvados), en sus respectivas poblaciones.

La tasa de costo-efectividad incremental * fue de $\$ 11.382$ cuando se comparó la colonoscopía con la SOMF. En el análisis de sensibilidad la búsqueda de SOMF es más sensible a cambios en las tasas de compliance que la colonoscopía. Distintos supuestos en cuanto a la sensibilidad y especificidad de las pruebas de SOMF, frecuencias en las estrategias, eficacia de la colonoscopía en la prevención del cáncer y la incidencia de pólipos tienen menor influencia sobre la diferencia de la costo-efectividad entre la colonoscopía y la búsqueda de SOMF.

\section{Conclusiones}

La colonoscopía representa el método más costo-efectivo en el screening del cáncer colorrectal porque reduce la mortalidad con un incremento en el costo relativamente bajo.

\section{Comentario}

El cáncer colorrectal es una enfermedad que tiene desde un punto de vista epidemiológico un fuerte impacto en la comunidad. En Argentina, de acuerdo a los datos procedentes del Ministerio de Salud de la Nación del año 1998, el cáncer colorrectal es la segunda causa de muerte por tumores malignos y es responsable de 5300 defunciones anuales. La probabilidad de desarrollar cáncer colorrectal a través de toda la vida y en ambos sexos es de un $6 \%$ (1 en 18).

Alrededor del $90 \%$ de los cánceres colorrectales se desarrollan en personas de más de 50 años de edad con un pico de incidencia entre los 65 años y los 75 años. Si el diagnóstico se realiza en la forma habitual, el $65 \%$ de los pacientes presenta enfermedad avanzada localmente 0 a distancia y la sobrevida global a 5 años se ubica entre el $50 \%$ y el $60 \%$. Sin embargo, el pronóstico varía radicalmente cuando el diagnóstico es temprano, con sobrevidas a los 5 años del $80 \%$ en el cáncer del recto y del $90 \%$ en el cáncer de colon.

Lesiones premalignas (pólipos adenomatosos) preceden a más del $90 \%$ de los cánceres colorrectales y su progresión resulta de un proceso en etapas que dura varios años. Esta lenta progresión de adenoma a carcinoma, provee una ventana aceptable para la realización de un test adecuado que permita la detección de adenomas y su exéresis o al menos la detección de lesiones malignas en estadios tempranos.

En relación a los métodos de screening, la búsqueda de SOMF ha mostrado una reducción de la mortalidad máxima estimada del cáncér colorrectal del $45-50 \%$ en base a evidencias que surgen de ensayos clínicos controlados y aleatorizados. Por otro lado, la sigmoidoscopía flexible evidencia una reducción de la mortalidad del $50-60 \%$ en estudios caso control. Por último, la colonoscopía reduce la mortalidad en $75-80 \%$ de acuerdo a evidencias indirec tas que surgen de ensayos clínicos controlados en pacientes con SOMF positiva.

El $90 \%$ de los pólipos colorrectales son extirpables mediante colonoscopía ${ }^{1}$, razón por la cual este estudio endoscópico resulta el método más poderoso para disminuir la incidencia del cáncer colorrectal

Por último, es preciso remarcar que para decidir políticas de salud es necesario contar con evidencia rigurosa acerca de los beneficios, riesgos y costos de una intervención, así como también tener en cuenta los valores de la sociedad. Una herramienta más para ayudar a tomar estas decisiones es el análisis de costoefectividad, un método para evaluar y comparar distintas estrategias de salud con respecto no sólo a la efectividad de una intervención, sino también cuánto le cuesta a la sociedad esa cuota extra de efectividad ${ }^{2}$. En modelos de análisis de decisión el screening de cáncer colorectal mostró ser más costo-efectivo que el screening de cáncer de mama y el de cáncer de cuello uterino ${ }^{3}$.

Conclusiones del comentador: Este estudio mostró las ventajas potenciales de la estrategia con colonoscopía cada 10 años en el screening del cáncer colorrectal agregando nueva información que justifican a dicha intervención en comparación con la SOMF y la sigoideosopía.

Dr. Jorge Olmos [ Servicio de Gastroenterologia. Hospital Italiano de Buenos Aires ]

Dr. Mariano Marcolongo [ Servicio de Gastroenterologia Hospital Italiano de Buenos Aires ]

\section{Referencias}

1. Winawer SJ et Al Prevention of the colorectal cancer by colonoscopic polypectomy. The National Polyp study Workgroup. N Engl J Med 1993; 329: 1977-81 .

2. Liberman DA. Cost-effectiveness model of colorectal cancer. Gastroenterology 1995; 109: 1781-90.

3. Sonnenberg FA et Al. Markov models in the medical decision making: a practice guide. Med Decis Making 1993; 13: $322-28$ 